\title{
Suitability assessments for advanced composite- metal hybrid material systems in automotive crash structural applications
}

\section{Michael Dlugosch* and Maximilian Volk}

Department of Material Dynamics,

Fraunhofer Institute for High-Speed Dynamics,

Ernst-Mach-Institut, EMI,

Eckerstrasse 4, 79104 Freiburg, Germany

Email: Michael.dlugosch@emi.fraunhofer.de

Email: Maximilian.Volk@emi.fraunhofer.de

*Corresponding author

\section{Dirk Lukaszewicz}

Vehicle Safety,

BMW Group, 80788 Munich, Germany

Email: Dirk.Lukaszewicz@bmw.de

\section{Jens Fritsch}

Department of Material Dynamics,

Fraunhofer Institute for High-Speed Dynamics,

Ernst-Mach-Institut, EMI,

Eckerstrasse 4, 79104 Freiburg, Germany

Email: Jens.Fritsch@emi.fraunhofer.de

\section{Stefan Hiermaier}

Fraunhofer Institute for High-Speed Dynamics, Ernst-Mach-Institut, EMI,

Eckerstrasse 4, 79104 Freiburg, Germany

Email: Stefan.Hiermaier@emi.fraunhofer.de

\begin{abstract}
The application of hybrid material systems composed of advanced composites and metals in automotive crash structural applications holds significant potential in terms of lightweighting and functional improvements. This paper proposes a comprehensive methodology to assess the suitability of vehicle body components for the application of hybrid material systems by analysing superimposed numerical crash simulation data of conventional steel bodies-in-white. The loading anisotropy and the global deformation are presented as two suitability criteria including an evaluation methodology to eventually select suitable hybrid material systems in the transition from
\end{abstract}


conventional to enhanced material employment and vehicle structure design. Additionally, the methodology provides novel insights into the global structural loading by simultaneously considering multiple crash load cases.

Keywords: automotive; crash structures; hybrid material systems; material selection, suitability analysis.

Reference to this paper should be made as follows: Dlugosch, M., Volk, M., Lukaszewicz, D., Fritsch, J. and Hiermaier, S. (2017) 'Suitability assessments for advanced composite-metal hybrid material systems in automotive crash structural applications', Int. J. Automotive Composites, Vol. 3, No. 1, pp.14-28.

Biographical notes: Michael Dlugosch is a Mechanical Engineer and $\mathrm{PhD}$ Candidate. His main areas of research are vehicle crashworthiness, multi-material systems and lightweight design.

Maximilian Volk is an Engineering Student and a Research Assistant in the field of numerical loading analysis of vehicle crash structures.

Dirk Lukaszewicz is a Senior Scientist in the fields of advanced composite materials and structures, automotive crashworthiness and passive safety.

Jens Fritsch is a Physicist and Head of the Department of Material Dynamics and the Fraunhofer EMI. His main areas of research are numerical and experimental methods in the field of automotive safety.

Stefan Hiermaier is the Director of the Fraunhofer EMI, University Professor and a renowned Researcher in the fields of impact physics, material and structural dynamics and sustainable systems engineering.

This paper is a revised and expanded version of a paper entitled 'New method to analyze numerical crash simulation data to assess the suitability of hybrid composite structures in automotive applications', presented at the American Society for Composite Materials' $30^{\text {th }}$ Technical Meeting, September 28-30, 2015, East Lansing, Michigan, USA.

\section{Introduction}

Growingly stringent regulations for $\mathrm{CO}_{2}$ emissions (European Union, 2009) and sustainability goals of car manufacturers motivate an increased effort in the exploration of new and effective lightweight design strategies. Since the load-carrying structures in the body-in-white (BiW) comprise roughly $25 \%$ (Leohold, 2011) of the entire vehicle weight, these structures offer a comparably high potential for mass reductions.

Advanced composites like carbon or glass fibre-reinforced plastics exhibit weightspecific crashworthiness characteristics that, although strongly dependent on the composite's constituents and their arrangement, mostly outrival those of metals (Thornton, 1979; Friedrich, Kopp and Stieg, 2003; Drechsler et al., 2000). Metals in turn offer relatively cost-efficient solutions with well understood and stable energy absorbing mechanisms. Taking into account higher tolerances for weight reduction costs for alternatively driven vehicles (Lukaszewicz, 2013), combining fibre-reinforced plastics 
(FRPs) and metals into hybrid material systems with two discrete material phases could open new possibilities in terms of weight-specific functional performance under crash. These new developments are part of a general trend of material diversification and specialisation in modern BiWs (Friedrich et al., 2008; Eickenbusch and Krauss, 2013), where each material systems' properties are supposed to suit the specific loading situation in the respective structural application.

In the field of material selection, many authors have proposed methods which aim to yield the best compromise among various - sometimes contradicting - selection criteria according to the given preconditions in the structural application. One popular approach has been proposed by Ashby (2005) defining a four-step screening process which combines the consideration of the design requirements and a documentation of the materials' properties. Other authors such as Chiner (1988) proposed modified versions of this scheme by combining or splitting the selection steps and putting emphasis on certain individual steps. Reuter (2014) condensed these ideas by setting up the four-step approach as depicted in Figure 1.

Figure 1 Material selection process

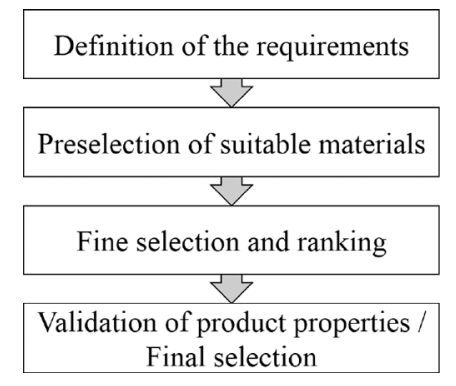

Source: Reuter et al.

The preselection and fine selection steps in this method are based on the evaluation of several criteria. One classical approach to this evaluation has also been proposed by Ashby (2009). This method is based on visual representation and is thus limited to a maximum selection of three parameters to be considered at the same time. Other methods such as the analytical hierarchy process (AHP) aim to evaluate these criteria in a specific order and to assign weighing factors in order to yield different alternative solutions with individual sets of parameters and a ranking value based on the evaluation results and the respective weighing factors for all criteria. Hambali et al. (2010) have successfully applied the AHP to the material selection for an automotive bumper structure and yielded a weight reduction by $26 \%$ compared to the reference system. In the context of automotive BiW material selection and criteria definition, Sahr (2011) has proposed a method to superimpose physical requirements and material properties based on six criteria such as the energy absorption capability, the structural integrity and stiffness in order to make a final material selection. Although the method successfully leads to a proper selection the dependency on expert knowledge to define requirements limits the flexibility and objectivity of the method. A methodology proposed by Kellner (2013) to assess the suitability and lightweight potential of different hybrid material systems for $\mathrm{BiW}$-structural applications is facing the same limitations. The requirement definition 
partly based on expertise of engineers compromises the transparency and reproducibility of the method. A suitability criterion for composite materials in BiW-applications based on the loading anisotropy has been introduced by Durst (2008). By comparing the principal stresses in a 2D-shell finite element (FE) for several load cases, Durst qualifies the overall (superimposed) anisotropy of the mechanical loading and produces a component value by computing its arithmetic mean. After visualising and colour coding this value, components with a rather anisotropic loading can be identified and considered as generally suitable to the application of fibre composite materials. Although this is a very innovative method, the formulation of this anisotropy criterion - especially for entire components - as presented lacks robustness and accuracy.

The aim of this novel approach is to define criteria and an evaluation strategy particularly applicable to make suitability assessments for FRP-metal hybrid material systems. Based on the existing approaches, this new method aims to generate these assessments by utilising criteria which are defined through the analysis of experimental results obtained by the authors (Dlugosch et al., 2015; Dlugosch et al., 2016) (Figure 2 exemplarily depicts a tested hybrid component) and requirements resulting from the evaluation of superimposed crash loadings in a conventional (metallic) vehicle structure. Consequently, this method tries to enable the transition from conventional vehicle structures employing conventional BiW-materials towards lightweight structural concepts composed of hybrid material systems.

Figure 2 Example of a CFRP-steel hybrid component tested under axial impact in a previous study conducted by the authors

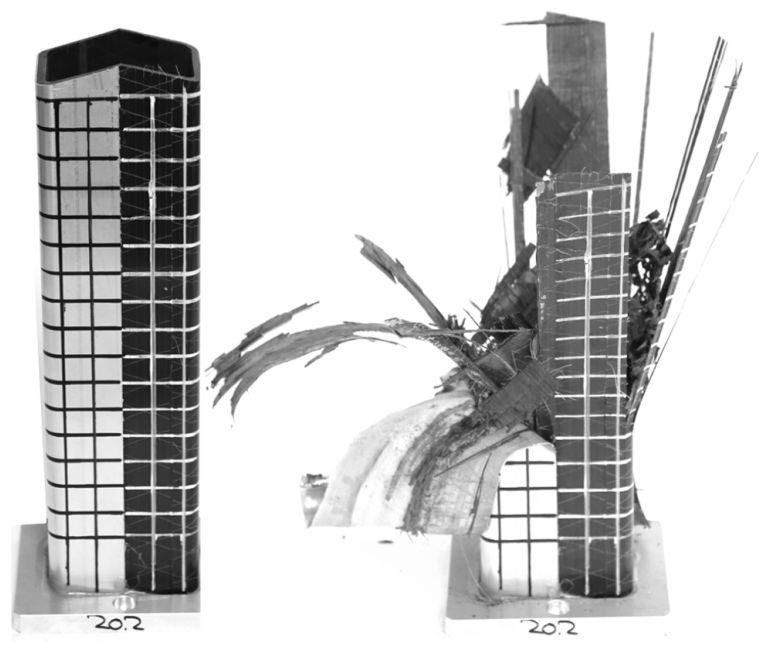

Source: Dlugosch et al., 2016

Further aims are to generate new insights into the crash loading of a BiW by visualising the results of this loading analysis and to identify design principles that have so far mostly been qualitatively and subjectively expressed through engineering expertise. 


\section{Methodology overview}

This chapter will provide an overview of the presented methodology and an abstract description of its technical implementation. It is noted that this paper focusses on the creation of loading profiles based on full vehicle FE crash simulations expressed through criteria which then can be used to qualify the suitability of certain hybrid material systems. This method has a framework which is open to further enhancements and extensions which also allows for the transfer of the method to other types of structural applications or material systems.

\subsection{Concept of the methodology}

As derived earlier, the method presented is based on existing theories dealing with material selection based on the comparison between the property profiles of the available material systems and the requirements within the structural application. This comparison is made possible by defining suitable criteria which can be assigned a value for both the material properties and the structural requirements. As a part of a holistic approach to the design of automotive crash structures composed of hybrid FRP-metal material systems, the authors have broadly tested these material systems experimentally to define property profiles to be compared with loading profiles of the relevant structures (Dlugosch et al., 2016; Dlugosch et al., 2015). These hybrid material systems are mostly compositeintensive systems composed of a prepreg-based composite structure reinforced with adhesively bonded metal sheets after curing.

In order to properly define the mechanical requirements resulting from a specific structural application and the crash loads, this structure has to bear (loading profile), this method considers the evaluation of the mechanical loads (e.g. stresses) and the structural responses (e.g. deformations). Designing structures with tailored properties with respect to specific loads often implies a dramatic reduction in the performance when subject to a modified loading situation (robustness). This is particularly true for the design of structures involving composite materials. Considering this, this novel approach uses the information of all significant crash load cases and condenses them into a global, superimposed loading profile.

\subsection{Technical implementation}

As stated earlier, the loading profile of an automotive structure is based on physical data stored in full vehicle FE-simulation models. The software used to model and run the full vehicle crash simulation is Abaqus/Explicit by Simulia (Dassault Systèmes). The simulation of each load case yields one result file called output database file (.odb-file), which contains the model geometry and all requested field and history outputs such as the stress or strain information. Thanks to a scripting interface, these odb-files can be accessed and modified using Python scripts. The information flow while superimposing the relevant load cases for a loading profile is schematically depicted in Figure 3. 
Figure 3 Schematic representation of the information flow

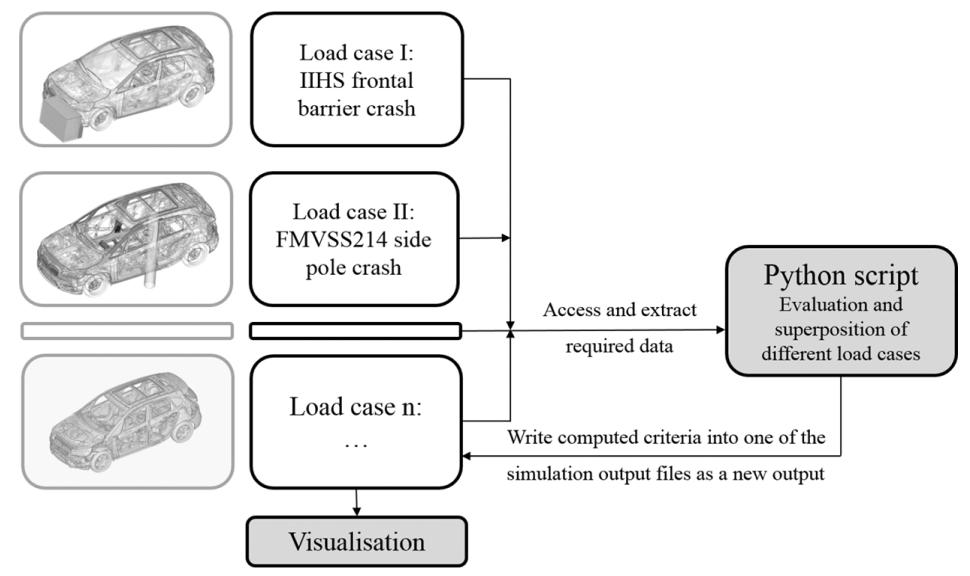

All .odb-files are consecutively accessed by Python scripting for the relevant data to be read and extracted. The data of all load cases are then temporarily stored and processed in the Python script before being written back into one of the odb-files as new outputs which in turn can then be conveniently visualised using common post-processing software, such as the Abaqus Viewer.

\section{Suitability criteria}

This chapter proposes two criteria used to qualify the conformity of the loading profile and the property profile. As indicated, this method offers the flexibility to be enhanced and expanded by the definition of further criteria. The authors would like to note that all figures have been derived from coloured illustrations. Although the greyscale values have been individually adjusted, a full interpretation partly needs to be supported by the framing text sections.

\subsection{Suitability criterion: plastic deformation and energy absorption}

One important criterion for the evaluation of a crash structure's loading situation is the amount of plastic deformation which is often directly linked to the level of energy absorption. The amount of plastic deformation thus gives an indication of the structure's functional role in the crash management of a vehicle's structural subsystem. The value considered here is the equivalent plastic strain (PEEQ) (Dassault Systemes) defined as

$$
\text { PEEQ }=\sqrt{\frac{2}{3} \cdot \varepsilon_{i j}^{\mathrm{pl}} \cdot \varepsilon_{\mathrm{ji}}^{\mathrm{pl}}} .
$$

In order to assess the overall plastic deformation considering all important load cases, the PEEQ value of each shell element in the vehicle (sub-) model for each load case in the 
last time frame is stored in an element data array. After reading all odb-files and processing the data, the largest value in the stored array of each element is written back as the newly generated output. Figure 4 depicts the results based on this method.

Figure 4 Superposition of the plastic deformation output in a bumper of two frontal crash load cases

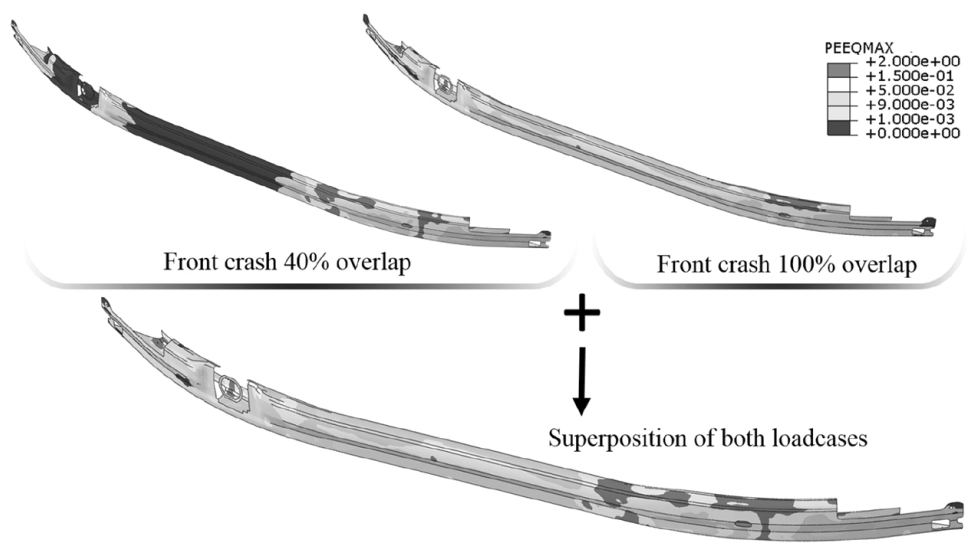

The $40 \%$ overlap crash has a significant maximum of deformation on the right side (reader's perspective) of the bumper and almost no plastic deformation on the left side. The 100\%-overlap crash PEEQ results appear more balanced, which is then expressed in the depiction of the superimposed PEEQ values. Applying this evaluation to a full BiW model results in a rather complex representation which might be difficult to interpret as depicted on the left side of Figure 5.

Figure 5 Element-wise (left) and component-wise (right) representation of the plastic deformation for single and superimposed frontal crash load cases
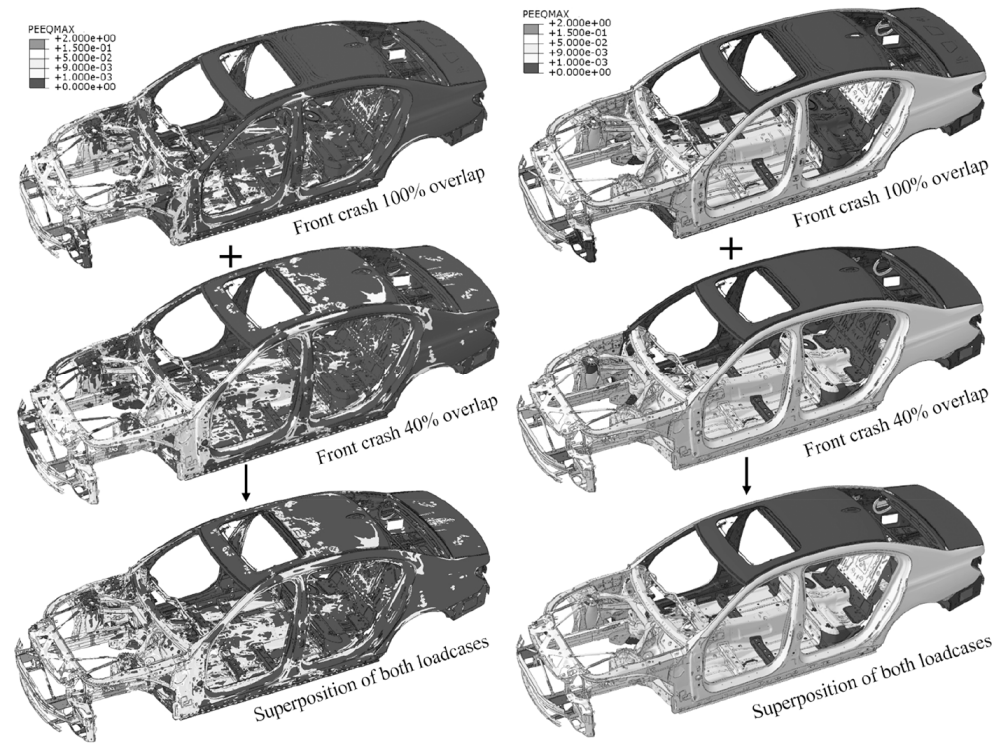
For a more convenient representation, a mean plastic deformation is introduced, which is defined as the sum of all PEEQ values for all elements in a component divided by its number of elements. This simplified representation allows to easily identify components that are subject to a certain amount of mean plastic deformation. The shading of the side frame as one component indicates the difficulty inherent in such generalisations. While the front of the component is strongly deformed, the back is clearly not significantly deformed. Still, the mean value assigned to the entire component indicates a 'deformed component' (compare to rear and roof shading). This smearing of values and its advantage of a convenient interpretation might thus only be a first step in such an analysis.

\subsection{Suitability criterion: loading anisotropy}

As previously introduced by Durst (2008), the loading anisotropy can be considered an indication for the suitability of composite materials. Since the authors experimentally confirmed the general applicability of the rule of mixture (Dlugosch et al., 2015) for the hybrid material systems covered in this study, an anisotropic loading could thus be considered a suitability criterion for a rather composite-intensive hybrid system, where the 'hydrostatic' share of the stress is carried by the metal phase and the deviatory share is carried by the highly anisotropic composite materials.

The anisotropy evaluation is based on the principal stresses of the $2 \mathrm{D}$ stress tensor in a shell element, which are calculated through a coordinate system rotation by the principal angle $\varphi_{0}$ (Figure 6 , left). In the design optimisation of composite structures and especially in tailored fibre placement processes, the principal stresses and directions are commonly used as fibre orientation angles in order to minimise shear loadings of the fibres (Crothers et al., 1997). A state of stress here is considered anisotropic if one principal stress is significantly larger than the other one, see Figure 6 (right).

Figure 6 Rotation of the coordinate system to calculate the principal stresses (left) and the schematic depiction of the anisotropy definition based on the principal stresses (right)
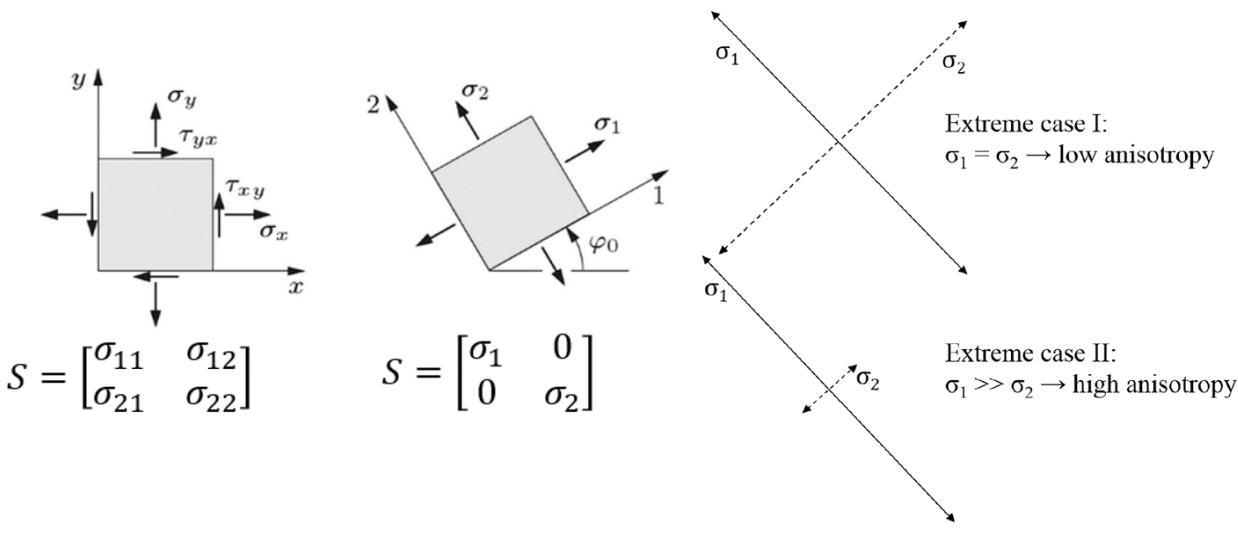
For the evaluation of a superimposed anisotropy criterion for an element, a new calculation method of increased robustness (compared to the method proposed by Durst) is introduced. By additively superimposing the stress tensors of the considered load cases and then calculating the principal stresses and their directions, no further weighing factors are necessary and the number of calculation steps could be reduced, which is of major importance regarding the amount of elements to be computed (a common BiWmodel comprises roughly one million elements). The principal stress factor (PSF) is thus defined as

$$
\operatorname{PSF}=\frac{\operatorname{Max}\left(\left|\sigma_{1}\right|,\left|\sigma_{2}\right|\right)-\operatorname{Min}\left(\left|\sigma_{1}\right|,\left|\sigma_{2}\right|\right)}{\operatorname{Max}\left(\left|\sigma_{1}\right|,\left|\sigma_{2}\right|\right)},
$$

where $\sigma_{1}$ and $\sigma_{2}$ are the principal stresses based on the superimposed stress tensor. Only absolute values are considered here, which implies that tension and pressure loadings are treated equally. This approach is based on the previous findings by the authors (Ihle, 2016) and acknowledged methods, such as the classical lamination theory (Schürmann, 2007). The PSF has a value range between 0 (isotropic) and 1 (highly anisotropic).

Analysing the PSF for a single front crash load case and visualising it including the vector of the first principal stress (which can be considered the most beneficial fibre inclination angle phi in the element location (Rettenwander, Fischlschweiger and Steinbichler, 2014)), as depicted on the left side in Figure 7, yields an interesting representation of the anisotropically loaded areas in a structure.

Figure 7 Evaluation of the PSF for a single barrier front crash with $40 \%$ overlap in the door sill area (left) and the schematic depiction of two extreme cases of low and high uniformity (right)

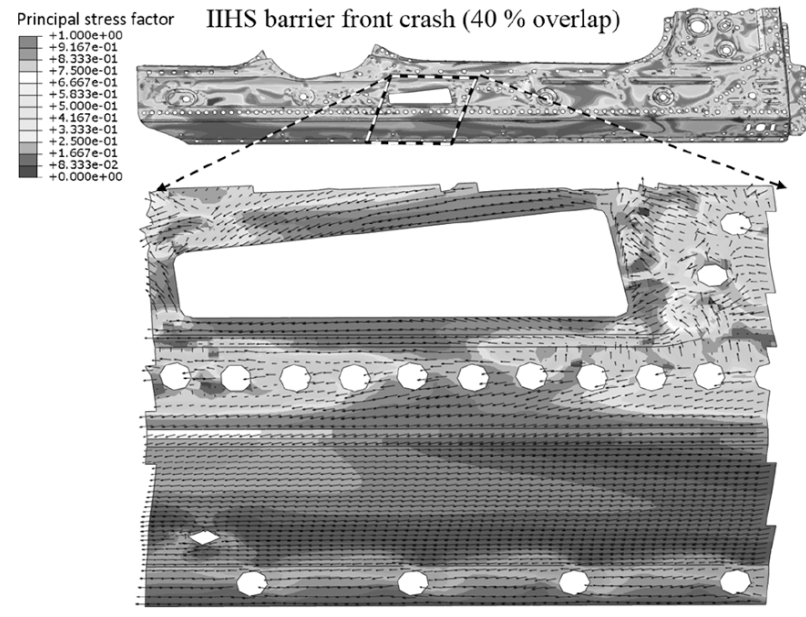

Extreme case I:

Irregular directions $\rightarrow$ low uniformity

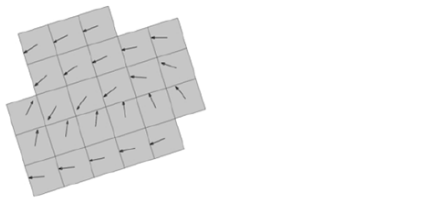

Extreme case II:

Regular directions $\rightarrow$ high uniformity

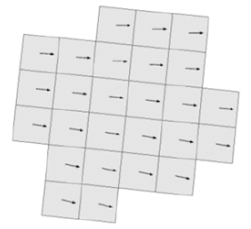


Some areas such as those with a high density of spot welds are quite isotropic due to the complex loading situation in the surrounding of joints. Clearly, in the lower left picture of Figure 7, there are some areas that show a high anisotropy (dark shading) and exhibit a uniformly distributed orientation of the maximum principal directions. Both are important when trying to qualify the anisotropy of a structure - not only of an element. This fact is considered by defining a uniformity index, which qualifies the uniformity of the principal angles' orientation within a component. The right picture in Figure 7 depicts two cases of neighbouring elements with an indication of their maximum principal direction.

This uniformity index of a component (or element set) $U$ is defined as

$$
U=\max \left(1-\left(\frac{\sum_{i=1}^{n}\left|\alpha_{\mathrm{ref}}-\alpha_{\text {Element }}\right|}{45 \cdot n}\right)^{m},\right.
$$

where $\alpha_{\text {ref }}$ is a reference angle in the global coordinate system, which is chosen to yield the minimum sum of deviations implying a maximum uniformity. The number of elements is denoted by $n$ and $m$ is a sensitivity exponent, which can be used to adjust the behaviour of $U$ in terms of convergence towards 1 (maximum uniformity). The angle $\alpha_{\text {Element }}$ is a conditioned direction of the respective element's maximum principal stress. The denominator comprises a normalisation including the factor 45 , which represents the maximum deviation of $45^{\circ}$.

As mentioned above, in order to qualify a component's loading anisotropy, it is essential to evaluate both the stress anisotropy and the uniformity of the orientation of the maximum principal stress in the entire component. This is accounted for when considering the component anisotropy value AnIso defined as the mean value of all elements' PSF values multiplied by the component's uniformity index $U$ expressed as

$$
\text { AnIso }=\frac{\sum_{i=1}^{n} \mathrm{PSF}_{i}}{n} \cdot U .
$$

Since both the PSF and the uniformity index $U$ have a normalised value range between 0 and 1 , the AnIso values also have the same range, providing for a reasonable way of interpretation. A superimposed evaluation of the component anisotropy AnIso in a BiW subject to a frontal barrier crash and a side pole crash is depicted in Figure 8. 
Figure 8 Evaluation of the component anisotropy value AnIso for two individual load cases and their superposition

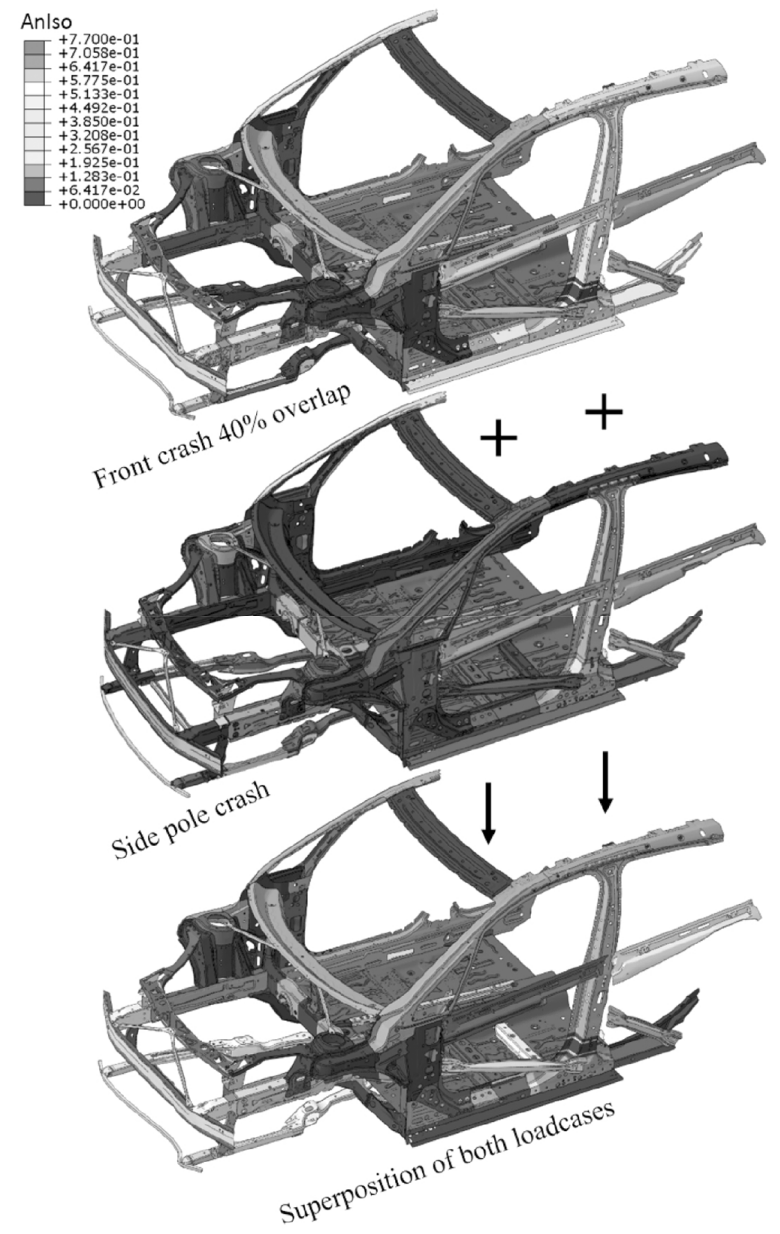

Looking at the topmost picture in Figure 8, the AnIso value evaluated for a $40 \%$-overlap frontal barrier crash (IIHS standard) load case accentuates load path and lattice structures such as the frontal longitudinal members (with an emphasis on the overlapped left side), the door sills, pillars and struts. When superimposing both load cases (lowermost picture), rather low AnIso values of the side pole evaluation generally demagnify those of the first load case. This is particularly observed at the door sill, which exhibits a significant decrease in the AnIso value for the superimposed data compared to the front crash data. This emphasises the necessity to superimpose data in order to robustly define anisotropically loaded structural areas. Although this method is already fully applicable to real models in serial development, the light grey coloured components of the superimposed case in Figure 8 indicate that some minor technical hurdles - such as inconsistent element IDs - still need to be overcome. 


\section{Evaluation methodology}

In order to yield a consistent, reproducible suitability assessment based on the structure's loading and the hybrid material systems' property profile, an evaluation methodology is proposed. For reasons of scope and according to the criteria presented a subset of the entire methodology is introduced in this paper. Figure 9 schematically depicts the evaluation methodology.

Figure 9 Subset of the suitability evaluation methodology based on the analysis of the loading situation and the hybrid material systems' properties

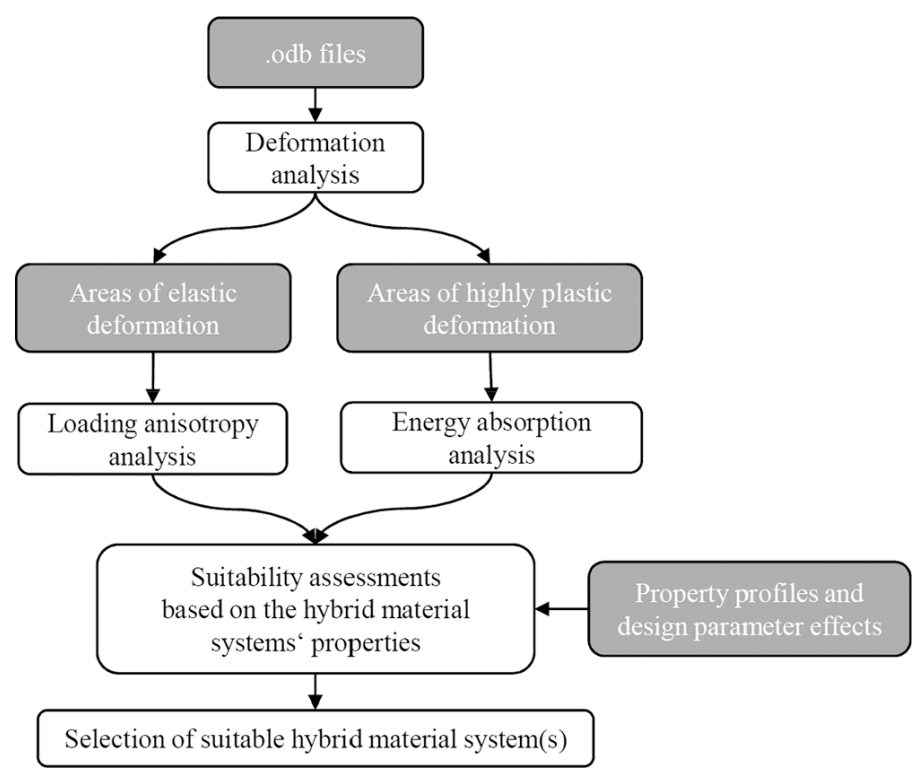

Since the anisotropy evaluation only yields reliable results in areas without plastic deformation, the deformation analysis is the first step after gathering the odb-files of the crash load cases to be considered. After that, two possible paths can be evaluated. In areas of pure elastic deformation, an anisotropy evaluation is performed, in areas of highly plastic deformation, an energy absorption analysis is executed. The definition of a suitable threshold needs to be adapted to the vehicle model and the crash load cases considered in the respective analyses. With the respective results, a hybrid material system can be selected according to its suitability based on its properties experimentally determined by the authors. The anisotropy criterion relates to the fibre layup and the cross-sectional ratio of advanced composite to steel. In highly plastically deformed areas, the energy absorption criterion can directly be compared to the experimental database and the related parameter effects. It is noted that process of the energy absorption analysis is not described here, since it is technically very close to the evaluation of plastic deformation. 


\section{Summary and discussion}

A new comprehensive methodology for the suitability assessment of advanced composite-metal hybrid material systems in automotive crash structures has been presented. Two criteria and a respective evaluation methodology have been proposed, which allow for the identification of elastically and highly plastically deformed automotive structures based on superimposed FE full vehicle crash simulations. Elastically deformed structural parts are analysed with respect to their mechanical loading anisotropy based on principal stresses and their directions of the superimposed stress tensors. The level of loading anisotropy can then be used to qualify the general suitability of FRP hybrid systems and to indicate the cross-sectional share of FRP and its fibre layup. A direct superposition of the stress tensors significantly increases the robustness and computational performance compared to the previous proposals. Highly plastically deformed structures are assumed to take the role of an energy absorbing structure providing a criterion to qualify the suitability of hybrid material systems based on their energy absorption capabilities. Linking the criteria in the evaluation methodology to the experimental database (including the effects of major design parameters) previously established by the authors allows for a general suitability assessment of various hybrid material systems and a final selection.

Additionally, it is possible to provide new insights into the global loading situation of a vehicle by simultaneously considering a number of crash load cases through visualisation in a common post-processing software. Figure 10 depicts a simplified result visualisation of the anisotropy and the deformation analysis of superimposed crash load cases.

Figure 10 Global anisotropy and deformation analysis of superimposed side pole (FMVSS 214 standard) and front barrier ( $40 \%$ overlap, IIHS standard) full vehicle crash simulation results

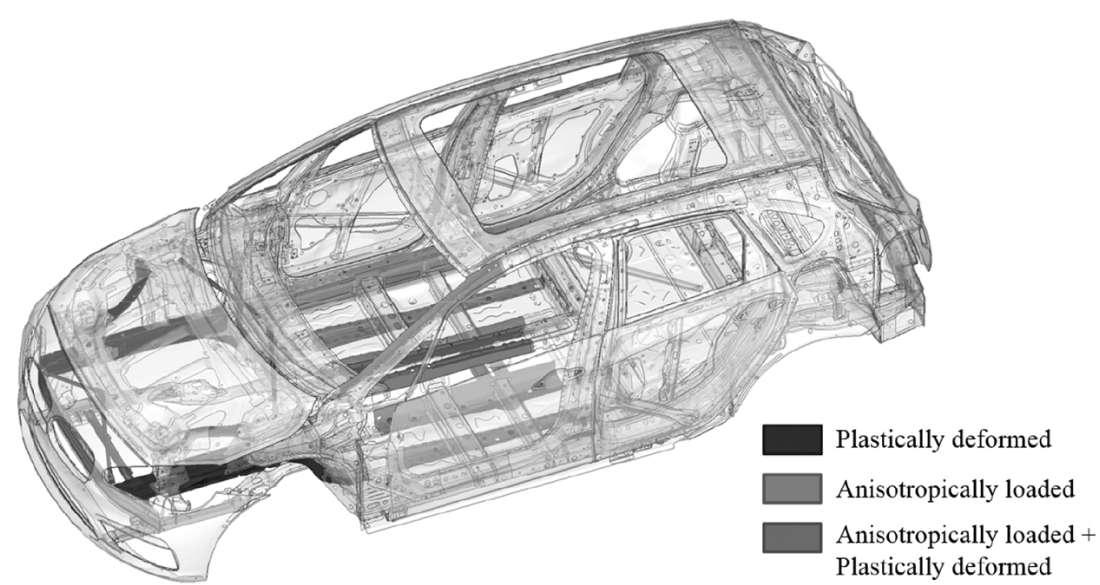

Selected components are highlighted and shaded according to their significant loading data in terms of deformation and anisotropy. This figure suggests that engineering design principles - such as structural role assignment (e.g. energy absorption) and load path design (see subfloor structures) - might be 'decoded' with the further development and enhancement of this method. This is of significant importance to the new method since it establishes the flexibility and objectivity often lacked in previously introduced methods. 
Although the criteria presented provide a reasonable and easily applicable way of performing suitability analyses, further criteria need to be defined in order to subsequently narrow down the selection of suitable hybrid material systems. These new criteria could then also be used to expand the evaluation methodology from its current state. Although some ideas are already implemented, the gap between purely elastic and significant plastic deformation needs further improvement.

Finally, this method needs to be validated by implementing some suitable material suggestions and virtually or experimentally testing the structures.

Future works might include the extension of this methodology to be used in a design optimisation process, which comprises the interaction between structural design and the application of different material systems. Significant tasks would be to define criteria that can be evaluated irrespective of the original type of material system and to include the framework presented into an optimisation workflow.

\section{Acknowledgements}

The authors would like to express their gratitude to their partners and colleagues at the BMW Group and Fraunhofer EMI for valuable discussions and support.

\section{References}

Ashby, M.F. (2005) Materials Selection in Mechanical Design, 3rd ed., Butterworth-Heinemann, Amsterdam, Boston.

Ashby, M.F. (2009) Granta CES Edupack 2009. 2 Material and Process Selection Charts. Available at: http://www.me.uprm.edu/vgoyal/inme4011/Online_inme4011/Topic2_MaterialSelection/ AshbyCharts.pdf (accessed 28 October 2013).

Chiner, M. (1988) 'Planning of expert systems for materials selection', Materials \& Design, Vol. 9, No. 4, pp.195-203.

Crothers, P.J., Drechsler, K., Feltin, D., Herszberg, I. and Kruckenberg, T. (1997) 'Tailored fibre placement to minimise stress concentrations', Composites Part A: Applied Science and Manufacturing, Vol. 28, No. 7, pp.619-625.

Dlugosch, M., Ihle, J., Lukaszewicz, D.H.-J.A., Fritsch, J. and Hiermaier, S. (2016) Experimental Investigation of Automotive Components Consisting of Hybrid FRP-metal Material Systems under Crash Loading, Paper presented at Euro Hybrid - Materials and Structures 2016, 20-21.04.2016, Kaiserslautern, Available at: http://eurohybrid.inventum.de/fileadmin/ Tagungen/2016/04-EuroHybrid/Grafiken/Euro-Hybrid_Proceedings_2016_small.pdf (accessed 15 May 2016)

Dlugosch, M., Lukaszewicz, D.H.-J.A., Fritsch, J. and Hiermaier, S. (2015) Experimental Characterization of Hybrid Material Systems Consisting of Sheet Metal and Advanced Composites, Paper presented at International Conference on Composite Materials, 19.-24.07, Copenhagen.

Drechsler, K., Heine, M., Mitschang, P., Baur, W., Gruber, U., Fischer, L., Öttinger, O., Heidenreich, B., Lützenburger, N. and Voggenreiter, H. (2000) 'Carbon fiber reinforced composites', in Ullmann's Encyclopaedia of Industrial Chemistry, Wiley-VCH Verlag GmbH \& Co. KGaA, Weinheim, Germany. http://onlinelibrary.wiley.com/doi/10.1002/14356007. m05_m02/full

Durst, K.G. (2008) Beitrag zur systematischen Bewertung der Eignung anisotroper Faserverbundwerkstoffe im Fahrzeugbau, Audi-Dissertationsreihe, Bd. 3, 1. Aufl, Cuvillier, Göttingen. 
Eickenbusch, H. and Krauss, O. (2013) Kohlenstofffaserverstärkte Kunststoffe im Fahrzeugbau Ressourceneffizienz und Technologien: Kurzanalyse Nr.3 und Dokumentation des Fachgesprächs, Berlin.

European Parliament, Council of the European Union: Regulation (EC) No 443/2009 of the European Parliament and the Council of 23 April 2009. In: Official Journal of the European Union, L 140 (2009-04-23), Nr. 52, S. 4. http://eur-lex.europa.eu/legal-content/EN/ TXT/?uri=CELEX:02009R0443-20130508 - Überprüfungsdatum 2016-10-18

Friedrich, H., Kopp, J. and Stieg, J. (2003) 'Composites on the way to structural automotive applications', Materials Science Forum, Vol. 426-432, pp.171-178.

Friedrich, H., Treffinger, P., Kopp, G. and Knäbel, H. (2008) 'Werkstoffe und Bauweisen ermöglichen neue Fahrzeugkonzepte', in Schindler, V. and Sievers, I. (Eds.): Forschung für das Auto von morgen: Aus Tradition entsteht Zukunft; mit 6 Tabellen, Springer, Berlin, pp.301-347.

Hambali, A., Sapuan, S.M., Ismail, N. and Nukman, Y. (2010) 'Material selection of polymeric composite automotive bumper beam using analytical hierarchy process', Journal of Central South University of Technology, Vol. 17, No. 2, pp.244-256.

Ihle, J. (2016) Experimentelle Untersuchungen an crashbelasteten Fahrzeugstrukturen in FVKMetall-Hybridbauweise, Diplomarbeit, University of Applied Sciences Kempten, Kempten.

Kellner, P. (2013) Zur systematischen Bewertung integrativer Leichtbau-Strukturkonzepte für biegebelastete Crashträger, 1., Aufl., Cuvillier Verl., Göttingen.

Leohold, J. (2011) Chancen und Grenzen für eine nachhaltigen FVK-Einsatz im Automobil, CCeV Automotive Forum 2011, Ingolstadt.

Lukaszewicz, D.H.-J.A. (2013) 'Automotive composite structures for crashworthiness', in Elmarakbi, A. (Ed.): Advanced Composite Materials for Automotive Applications, John Wiley \& Sons Ltd, Chichester, UK, pp.99-127.

Rettenwander, T., Fischlschweiger, M. and Steinbichler, G. (2014) 'Computational structural tailoring of continuous fibre reinforced polymer matrix composites by hybridisation of principal stress and thickness optimisation', Composite Structures, Vol. 108, pp.711-719.

Reuter, M. (2014) Methodik der Werkstoffauswahl: Der systematische Weg zum richtigen Material ; mit ... 27 Tabellen und einer Vielzahl nützlicher Internetlinks, 2., aktualisierte Aufl., Hanser, München.

Sahr, C. (2011) Methodische Vorgehensweise der Werkstoffauswahl für die Karosserieentwicklung in Multi-Material-Bauweise, Dissertation, ika - Institut für Kraftfahrzeuge, RWTH Aachen University, Aachen.

Schürmann, H. (2007) Konstruieren mit Faser-Kunststoff-Verbunden, VDI-Buch, 2., bearbeitete und erw. Aufl, Springer-Verlag, Berlin, Heidelberg.

Simulia (Dassault Systèmes) (2014): ABAQUS FEA, Finite Element Simulation, Providence, RI, USA: Simulia (Dassault Systèmes).

Thornton, P.H. (1979) 'Energy absorption in composite structures', Journal of Composite Materials, Vol. 13, No. 3, pp.247-262. 